\title{
Ancient polymorphisms contribute to genome-wide variation by long-term balancing selection and divergent sorting in Boechera stricta
}

Baosheng Wang ${ }^{1,2^{*}}$ D, Julius P. Mojica², Nadeesha Perera ${ }^{2}$, Cheng-Ruei Lee ${ }^{3}$, John T. Lovell ${ }^{4}$, Aditi Sharma ${ }^{5}$, Catherine Adam ${ }^{5}$ Anna Lipzen ${ }^{5}$, Kerrie Barry ${ }^{5}$, Daniel S. Rokhsar ${ }^{5}$, Jeremy Schmutz ${ }^{4,5}$ and Thomas Mitchell-Olds ${ }^{2^{*}}$

\begin{abstract}
Background: Genomic variation is widespread, and both neutral and selective processes can generate similar patterns in the genome. These processes are not mutually exclusive, so it is difficult to infer the evolutionary mechanisms that govern population and species divergence. Boechera stricta is a perennial relative of Arabidopsis thaliana native to largely undisturbed habitats with two geographic and ecologically divergent subspecies. Here, we delineate the evolutionary processes driving the genetic diversity and population differentiation in this species.

Results: Using whole-genome re-sequencing data from 517 B. stricta accessions, we identify four genetic groups that diverged around 30-180 thousand years ago, with long-term small effective population sizes and recent population expansion after the Last Glacial Maximum. We find three genomic regions with elevated nucleotide diversity, totaling about $10 \%$ of the genome. These three regions of elevated nucleotide diversity show excess of intermediate-frequency alleles, higher absolute divergence $\left(d_{X Y}\right)$, and lower relative divergence $\left(F_{S T}\right)$ than genomic background, and significant enrichment in immune-related genes, reflecting long-term balancing selection. Scattered across the genome, we also find regions with both high $F_{S T}$ and $d_{X Y}$ among the groups, termed $F_{S T}$ islands. Population genetic signatures indicate that $F_{S T}$-islands with elevated divergence, which have experienced directional selection, are derived from divergent sorting of ancient polymorphisms.

Conclusions: Our results suggest that long-term balancing selection on disease resistance genes may have maintained ancestral haplotypes across different geographical lineages, and unequal sorting of balanced polymorphisms may have generated genomic regions with elevated divergence. This study highlights the importance of ancestral balanced polymorphisms as crucial components of genome-wide variation.
\end{abstract}

Keywords: Genomic diversity, Ancestral polymorphism, Balancing selection, Speciation

\section{Background}

How evolutionary processes drive genetic divergence and eventually lead to speciation is a fundamental question in evolutionary biology [1]. Taking advantage of next-generation sequencing technologies, heterogeneous genomic variation has been documented in many species

\footnotetext{
* Correspondence: baosheng.wang@scbg.ac.cn; tmo1@duke.edu

${ }^{1}$ Key Laboratory of Plant Resources Conservation and Sustainable Utilization, South China Botanical Garden, Chinese Academy of Sciences, Guangzhou 510650, China

2Department of Biology, Duke University, Box 90338, Durham, NC 27708, USA Full list of author information is available at the end of the article
}

[2-8], but disentangling factors shaping genomic landscapes remain challenging [9].

Balancing selection maintains multiple advantageous polymorphisms in populations and increases genetic diversity [10]. In contrast, positive and purifying selection favor single advantageous alleles and reduce genetic diversity [11, 12]. Balancing selection can persist for many generations, and maintains ancient polymorphisms in nascent species pairs, resulting in genomic regions with increased nucleotide diversity $(\pi)$ in descendant species and low relative divergence $\left(F_{S T}\right)$ between species [13]. Alternatively, when

(c) The Author(s). 2019 Open Access This article is distributed under the terms of the Creative Commons Attribution 4.0 International License (http://creativecommons.org/licenses/by/4.0/), which permits unrestricted use, distribution, and reproduction in any medium, provided you give appropriate credit to the original author(s) and the source, provide a link to the Creative Commons license, and indicate if changes were made. The Creative Commons Public Domain Dedication waiver (http://creativecommons.org/publicdomain/zero/1.0/) applies to the data made available in this article, unless otherwise stated. 
selection varies geographically, it may favor locally adapted alleles in the nascent lineages [13]. In this case, ancestral balanced polymorphisms could be sorted unequally across descendant lineages by selection, generating genomic regions with both elevated $F_{S T}$ and absolute divergence $\left(d_{X Y}\right)$ $[3,7,13]$. Divergent sorting of ancient polymorphisms also could be facilitated by enhanced genetic drift as a consequence of population bottlenecks during speciation. Other processes can also generate highly differentiated regions (Table 1). During the process of isolation-with-migration, divergence might initiate in the regions with reduced gene flow and further extend to the surrounding areas due to the linked selection, resulting in genomic islands with elevated divergence (both $F_{S T}$ and $d_{X Y}$ ) $[3,8,11,13]$. Alternatively, forces such as background selection and recurrent selective sweeps tend to reduce genetic diversity in regions of low recombination, leading to elevated $F_{S T}$ but unchanged or decreased $d_{X Y}[2,4-6,11]$. These processes are difficult to discriminate because they are not mutually exclusive and could cause similar patterns in the genome (Table 1) [9]. Additionally, genetic drift and demographic processes may also be responsible for observed peaks of genomic diversity/divergence, mimicking the patterns produced by selection [14]. Therefore, inferring the evolutionary mechanisms that influence genomic landscapes requires detailed information on the speciation history and comparisons of lineages with contrasting divergence levels and geographic distribution [9].

Boechera stricta (Brassicaceae), a perennial relative of Arabidopsis thaliana, is native to largely undisturbed sites in western North America [15-17]. Previous studies identified two subspecies (EAST and WEST) of B. stricta, with further subdivision within the EAST subspecies [18-21]. While genetic variation within subspecies is driven by geographic isolation [20], the divergence between EAST and WEST is significantly influenced by ecological adaptation [20]. These subspecies occupy different habitats, and WESTERN genotypes are typically found in sites with more constant and abundant water supply, suggested that local water availability may be the selective force underlying ecological speciation between EAST and WEST subspecies [20]. In addition, greenhouse experiments revealed phenological and morphological traits under divergent selection between subspecies [19], and an EAST-WEST recombinant inbred line population segregates for many quantitative trait loci (QTLs) for ecologically important traits, including flowering time, herbivore resistance, fecundity, and lifetime fitness [21-24], which may have contributed to incipient ecological speciation in B. stricta. These characteristics, along with the sequenced genome [21], facilitate population genomic studies in B. stricta to understand how complex evolutionary forces drive divergence and speciation.

To understand how different evolutionary processes contribute to current genomic variation, we resequenced the whole genomes of 517 B. stricta accessions representing much of the species range. First, we investigated the population structure and history of species divergence. Next, we identified the signatures of long-term balancing selection influencing $\sim 10 \%$ of the genome. Finally, we looked for genomic regions that distinguish lineages and assessed the roles of different evolutionary processes in driving divergence. Our study provides an example for disentangling the multitude of evolutionary processes that may have shaped the patterns of genetic variation across the genome and improves our understanding of the cause and consequence of genomic divergence during speciation.

\section{Results and discussion}

We performed whole-genome resequencing of 517 inbred B. stricta accessions using Illumina Hiseq2000/ 2500 short-read technology (Additional file 1: Table S1). Raw reads were mapped to the $B$. stricta reference genome v1.2 [21]. After quality control, 484 accessions with mean depth $5.05 \times$ were retained for subsequent analyses (Fig. 1a; Additional file 1: Table S1). We called SNPs using HaplotypeCaller in GATK v3.8 [25] and applied a series of stringent filtering criteria to identify a total of 4 , 125,395 high-quality SNPs (see "Methods" section for

Table 1 Predicted characteristics of genomic islands of divergence under different evolutionary models

\begin{tabular}{|c|c|c|c|c|c|}
\hline Model & $F_{S T}$ & $d_{X Y}$ & $\begin{array}{l}\text { Polymorphism within } \\
\text { populations }\end{array}$ & $\begin{array}{l}\text { Local recombination } \\
\text { rate }\end{array}$ & $\begin{array}{l}\text { More islands } \\
\text { in sympatry }\end{array}$ \\
\hline $\begin{array}{l}\text { Reproductive isolation, divergence } \\
\text { with gene flow }[3,8,13]\end{array}$ & Elevated & Elevated & No prediction & No prediction & Yes \\
\hline $\begin{array}{l}\text { Local adaptation, divergence without } \\
\text { gene flow [3] }\end{array}$ & Elevated & Not elevated & Reduced & No prediction & No \\
\hline $\begin{array}{l}\text { Recurrent selective sweeps within } \\
\text { populations }[2,4-6,11]\end{array}$ & Elevated & Reduced & Reduced & Reduced & No \\
\hline $\begin{array}{l}\text { Background selection within } \\
\text { populations }[2,4-6,11]\end{array}$ & Elevated & Reduced & Reduced & Reduced & No \\
\hline $\begin{array}{l}\text { Sorting of ancestral balanced } \\
\text { polymorphisms }[3,7,13]\end{array}$ & Elevated & Elevated & No prediction & Unchanged or reduced & No \\
\hline
\end{tabular}




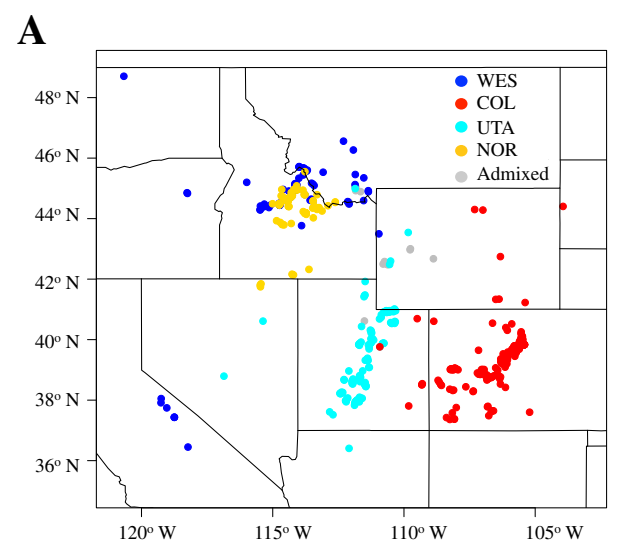

C

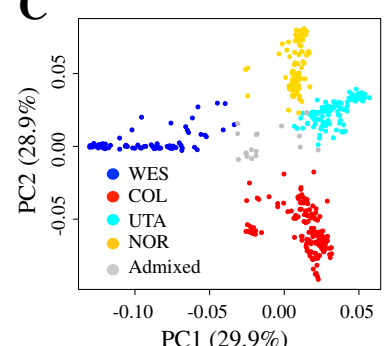

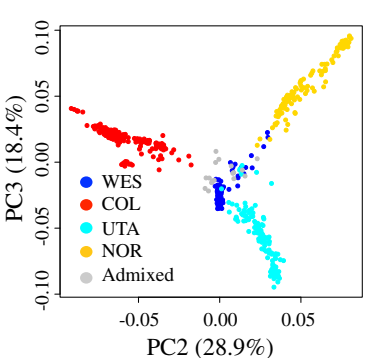

B
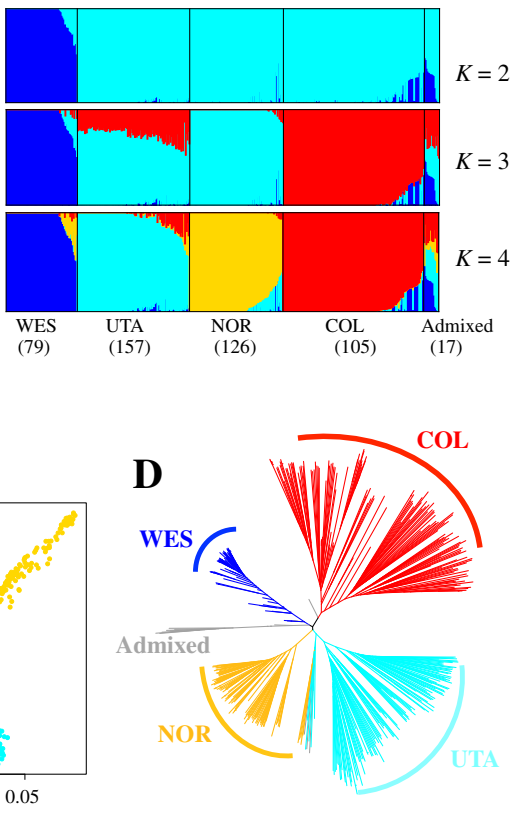

Fig. 1 Geographic distribution and population structure. a Geographic distribution of the 484 Boechera stricta accessions. Each individual was assigned into one of the four genetic groups, WES (blue), COL (red), UTA (light blue), and NOR (gold), as well as Admixed (gray). b Population structure of B. stricta inferred by Admixture. Each vertical bar represents an individual, with different colors representing one of the genetic ancestries. The number of individuals in each lineage is also shown. $K$ represents the number of structure groups for each analysis. $\mathbf{c}$ Genetic principal component analysis (PCA) of B. stricta based on genome-wide SNPs. Percent variation explained by each component is shown in parentheses. $\mathbf{d}$ Neighbor-joining (NJ) tree based on SNP data. Color scheme for genetic groups is the same in a-d

details). Comparison to Sanger sequences showed that the accuracy of our sequence calls exceeds $99.88 \%$ (Additional file 1: Table S2). To account for the uncertainty of genotypes called from short-read sequences, we estimated population genetic summary statistics based on genotype likelihoods as implemented in ANGSD [26].

\section{Discrete population structure and continuous genetic differentiation}

We used Admixture [27], FastSTRUCTURE [28], sNMF [29], and NGSadmix [30] to infer individual ancestry. These four methods gave very similar results (Fig. 1b; Additional file 1: Figure S1) and detected clear population structure in B. stricta. The mode with $K=3$ and 4 gave the highest $\Delta K$ values (Additional file 1: Figure $\mathrm{S} 2$ ); thus, we focus our analyses on the four genetic groups, WES, COL, UTA, and NOR (Fig. 1). With $K=$ 4 , we assigned each individual to one of the four groups if more than $50 \%$ of its genetic ancestry derived from the corresponding cluster (Fig. 1a; Additional file 1: Table S1). Seventeen individuals not matching this criterion were classified as "Admixed." The four genetic groups showed a clear geographical distribution pattern
(Fig. 1a). There is a little overlap among the groups, although NOR and WES have an area of sympatry in Montana and Idaho [21]. A principal component analysis (PCA) and a neighbor-joining (NJ) tree further confirmed the four genetic groups (Fig. 1c, d). High differentiation was detected among these four groups (Additional file 1: Table S3), similar to previous estimates based on microsatellite and low-copy nuclear DNA sequences $[17,18]$.

Genetic diversity in B. stricta consists not only of clusters, but also clines. Within groups, the geographic distribution of genetic variation is generally consistent with isolation by distance (IBD) models. We found significant correlations between pair-wise genetic distance and geographical distance within each group ( $r=0.19-0.36, P=0.0001-0.0023,10,000$ permutations in Mantel test; Additional file 1: Table S4), but the pattern of IBD varied among the groups (Additional file 1: Table S4), indicating different colonization or migration histories of these groups. In conclusion, we found both discrete population structure and continuous patterns of genetic differentiation in B. stricta; similar patterns have also been reported in humans [31] and A. thaliana [32]. 


\section{Population demography}

Recent divergence and long-term small effective population size of B. stricta

Estimates of demographic history and gene flow provide a sketch of population history and enable demographically informed simulations of population genetic variation under the assumption of selectively neutral evolution. Accordingly, we inferred the past demographic history of $B$. stricta from the joint site frequency spectrum (SFS) using coalescent simulation in fastsimcoal2 v.2.6.0.3 [33]. To avoid biases when determining the ancestral allelic states, we generated folded SFS following Excoffier et al. [33]. We used only fourfold degenerate sites and intergenic regions, as they are less affected by selection. Also, we removed the sites within the three genomic regions showing evidence of long-term balancing selection (see the "Balancing selection in B. stricta genomes" section). Fourteen demographic models were evaluated, considering a variety of scenarios for gene flow and population size changes (Additional file 1: Figure S3). The best-fit model (Model-11, Akaike's weight of evidence $\approx 1$, Additional file 1: Table S5) was a four-population isolationwith-migration model, where each group experienced two steps of population size changes after splitting (Fig. 2a; Additional file 1: Figure S4). By using a generation time of 2 years and mutation rate of $7 \times 10^{-9}$ substitutions per site per year [34], we estimated the model parameters and their associated $95 \%$ confidence intervals (CIs) based on 100 parametric bootstraps (Additional file 1: Table S6). Notably, mutation rate and generation time are difficult to estimate and may vary over space and time. Consequently, inferred times and population sizes would need to be revised if these estimates were inaccurate.

In this best-fit model, the estimated divergence time between the two subspecies (WES group and ancestor of three EAST subspecies groups) was $\sim 179$ thousand years ago (KYA; 95\% CI =154-185 KYA), and divergence within the EAST subspecies dates to 30-70 KYA (Fig. 2a; Additional file 1: Figure S4; Additional file 1: Table S6). Historical effective population size $\left(N_{\mathrm{e}}\right)$ was small in B. stricta; the estimated $N_{\mathrm{e}}$ values for the common ancestor of all groups, ancestor of EAST subspecies, and ancestor of UTA and NOR are $2.4 \times 10^{4}$, $3.6 \times 10^{4}$, and $3.9 \times 10^{4}$, respectively. After splitting, the WES and NOR groups both had small initial populations $\left(N_{\mathrm{e}}=1.9 \times 10^{4}\right.$ and $1.1 \times 10^{4}$, respectively $)$ comparable with their ancestors, while the COL and UTA groups had relatively larger initial populations $\left(N_{\mathrm{e}}=1.0 \times 10^{5}\right.$ and $1.1 \times 10^{5}$, respectively). The longterm small effective population sizes of WES and NOR are consistent with the relatively high level of linkage disequilibrium (LD) (Additional file 1: Figure S5) and low level of nucleotide diversity in these two groups (Additional file 1: Table S7). All groups experienced rapid population expansion at $\sim 12 \mathrm{KYA}$ and more recent exponential growth starting at $\sim 7.7 \mathrm{KYA}$ $(\mathrm{CI}=5.0-9.7 \quad \mathrm{KYA})$. Inferred gene flow was low among the groups (per generation migration rate $=$ $6.8 \times 10^{-10}-2.0 \times 10^{-5}$; Additional file 1: Table S6), which is expected given frequent inbreeding [17], low seed dispersal, and substantial geographic distances in B. stricta [15].
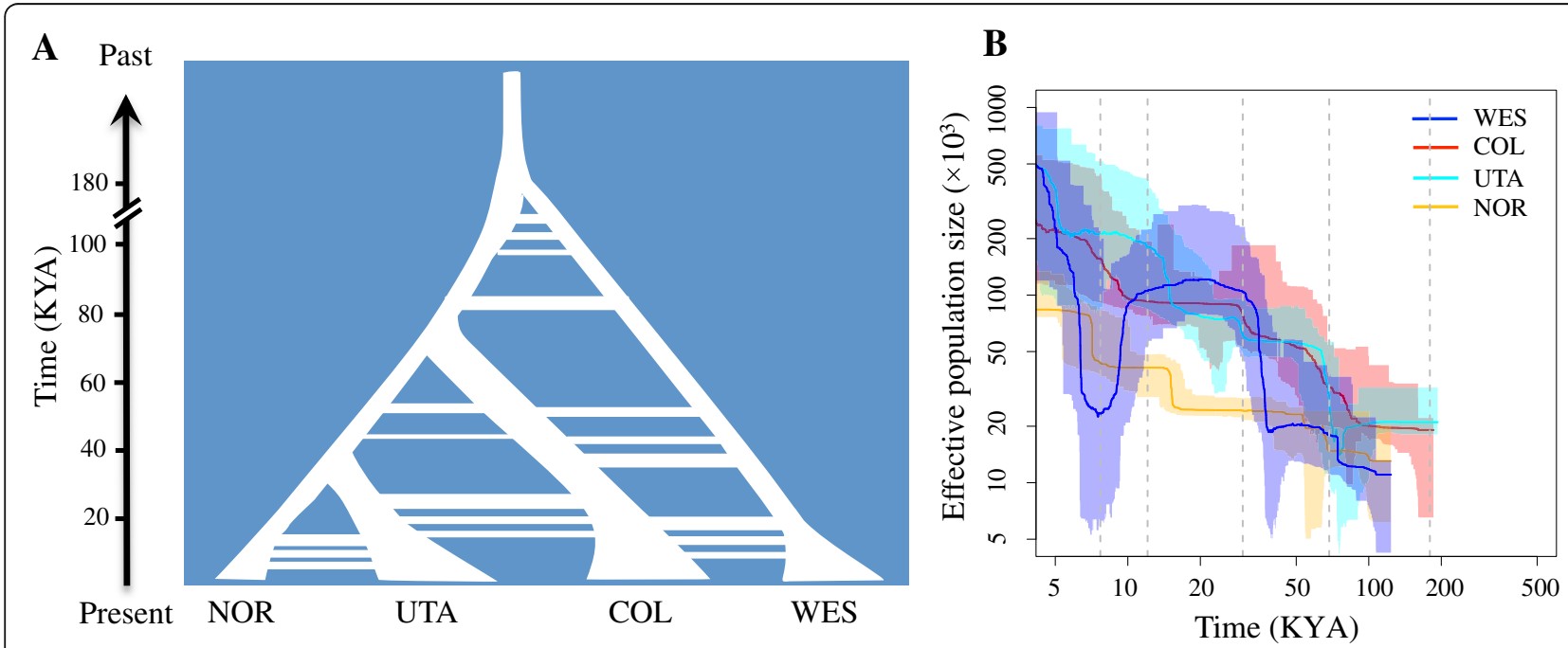

Fig. 2 Demographic histories of B. stricta. a Schematic representing divergence processes inferred by fastsimcoal2. Estimated divergence time, population size, and gene flow are indicated here and detailed in Additional file 1: Figure S4 and Tables S6. $\mathbf{b}$ Changes of effective population size through time in the four groups. Solid lines represent means, and shading represents $95 \%$ percentiles of estimates. Vertical dashed lines mark times of population divergence and expansion estimated by fastsimcoal2 


\section{Validation of demographic inference}

Evaluating null hypotheses of neutral evolution requires realistic population models, so we used two approaches to validate our demographic inferences. First, we evaluated the goodness-of-fit of the best model by comparing SFS and two summary statistics $\left(\pi\right.$ and $F_{S T}$ ) between observed and simulated data. We found that SFS and summary statistics predicted under neutrality are wellmatched to the data (Additional file 1: Figure S6). Second, to avoid limitations of model-based demographic inference, we also employed a model-flexible Stairway plot v.2 [35] method to investigate the recent fluctuation of $N_{\mathrm{e}}$, based on the folded SFS. In general, these results are consistent with those from fastsimcoal2. All groups showed lower ancestral population size $(1 \times$ $10^{4}-2 \times 10^{4}$ ) and recent population expansions within 15 KYA (Fig. 2b). In summary, validation analyses suggested that the best-fit model captures major aspects of the demographic history of our populations from patterns of genetic diversity. We applied this model in testing the significance of the outliers in subsequent analyses.

\section{Balancing selection in B. stricta genomes Long-term balancing selection affected $10 \%$ of the B. stricta genome}

In order to reveal evolutionary processes driving genomic variation, we estimated nucleotide diversity $(\pi)$ in $20-\mathrm{kb}$ non-overlapping windows across the genome. Then, we computed $Z$-transformations $(Z-\pi)$ separately in each group (see the "Methods" section for details). This transformation puts the four genetic groups on the same centered, relative scale of nucleotide diversity. Genomic regions with $Z-\pi \geq 2$ (corresponding to $4.30 \%$ of windows from all comparisons) were identified as outlier windows (Fig. 3). Within the four genetic groups, we detected $307-345$ outlier windows for $Z-\pi$ (“ $\pi$-islands"; Additional file 1: Table S7).
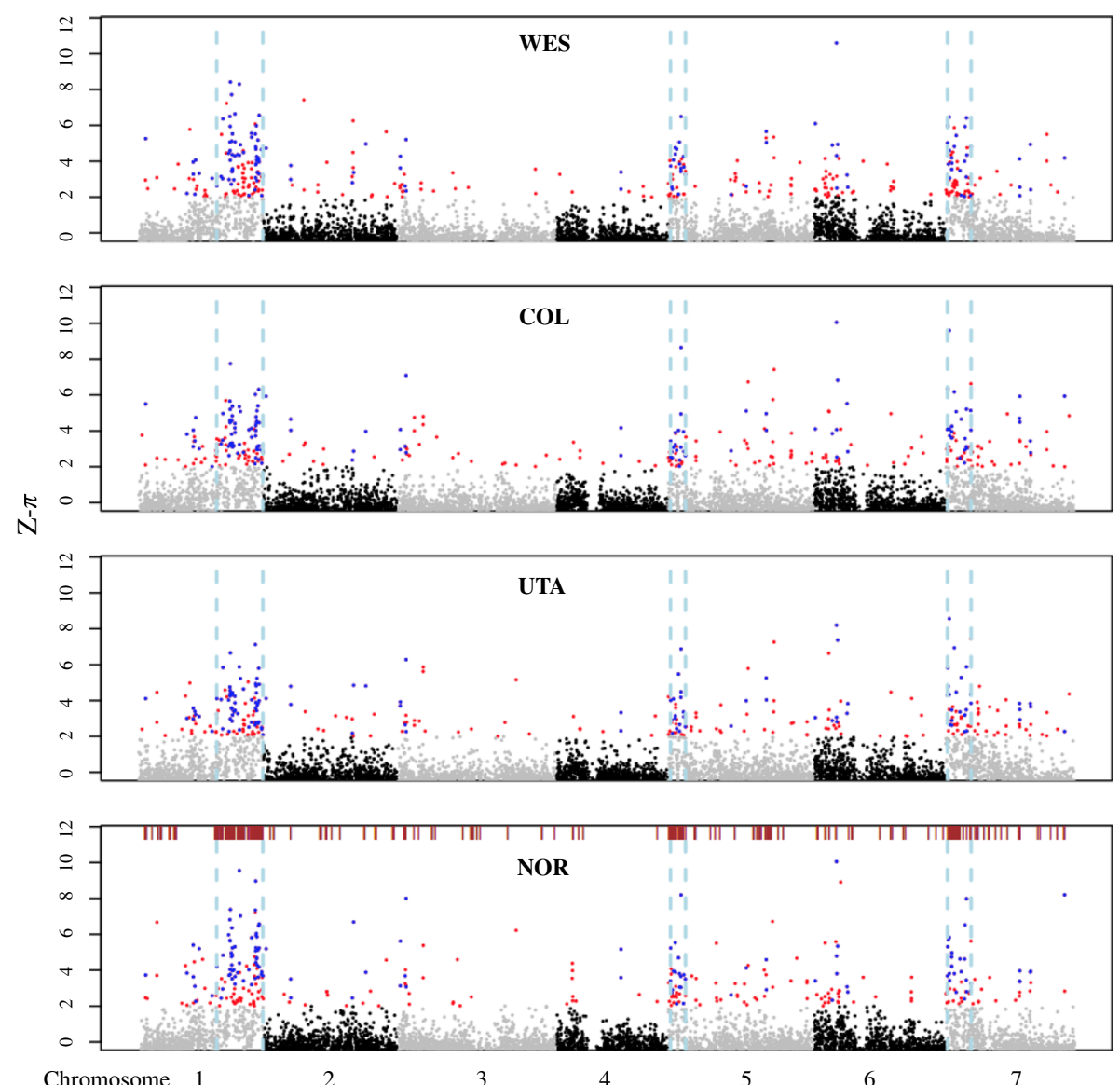

Fig. 3 Genome-wide pattern of nucleotide diversity. Z-transformed nucleotide diversity (Z- $\pi$ ) is plotted along the genome with 20-kb nonoverlapping windows for each group. The 130 outlier windows shared by all groups are shown in blue, and other islands specific to group(s) are in red. The dashed vertical lines (light blue) delimit three highly polymorphic genomic regions enriched for NBS-LRR genes. NBS-LRR genes are indicated by brown-colored bars above the Manhattan plot of NOR group. Alternating colors paint the different chromosomes, and windows with $Z-\pi<0$ are not shown 
Among these outlier windows, 130 were shared by all groups (henceforth referred to as shared outliers; Fig. 3), which could be generated by shared evolutionary forces influencing particular genomic regions. These $\pi$-islands were unevenly distributed in the genome and clustered into three genomic regions. These three regions were henceforth referred to as "Balancing selection (BLS) regions" (Fig. 3; see the "Methods" section for delimiting the BLS regions). BLS regions, with a total length of $20 \mathrm{Mb}$ ( $10 \%$ of the $196.5 \mathrm{Mb}$ assembled genome) [21], contain about half of the $\pi$-islands (46-49\%) including 91 (70\%) shared outliers (Additional file 1: Table S8).

All three BLS regions showed nucleotide diversity 2.57-3.88 times higher than the genomic background $\left(P<10^{-5}\right.$, randomization test; Additional file 1: Table S9) . Comparison of demographically informed neutral simulations with observed molecular variation suggests that non-neutral evolutionary processes have contributed to the elevated diversity in these regions (Additional file 1: Figure S7). The high diversity in BLS regions could be maintained by long-term balancing selection [10], but could also be due to the systematic variation in mutation rate, recombination rate, or gene density [12]. We checked each of these possible influences. First, to correct for variation in mutation rate among genomic regions, we divided diversity by divergence to an outgroup species, Boechera retrofracta $[21,36]$. These outgroupadjusted diversity levels are still higher in the BLS regions compared to genomic background $\left(P<10^{-5}\right.$, randomization test; Additional file 1: Table S9). Therefore, the high diversity in genomic islands is not attributable to the elevated mutation rates. Second, to assess the impact of recombination rate on genetic diversity, we estimated the population-scaled recombination rate $(\rho=$ $4 N_{\mathrm{e}} \mathrm{c}$ ) in $20-\mathrm{kb}$ windows across the genome using LDhelmet [37]. Following Wang et al. [4], we divided $\rho$ by genetic diversity $(\pi)$ to account for the confounding effects of local $N_{\mathrm{e}}$ and compared scaled $\rho(\rho / \pi)$ between the BLS regions and the rest of the genome. Relative to the genomic background, we found similar recombination rate in the BLS regions of groups WES and NOR (scaled $\rho: 0.25-0.29$ vs. $0.24, F_{3,7221}=1.03, P=0.377$ in WES; $0.16-0.26$ vs. $0.36, F_{3,6676}=2.42, P=0.064$ in NOR) but significant higher and lower recombination in the BLS regions of groups COL and UTA, respectively (scaled $\rho$ : $0.73-0.90$ vs. $1.15, F_{3,7198}=5.76, P=0.0006$ in UTA; $0.77-1.13$ vs. $0.59, F_{3,7287}=34.77, P<0.0001$ in COL; Additional file 1: Table S10). It is possible that other historical factors, such as population structure, might explain the patterns in COL and UTA. For example, we estimated the recombination rate in each subgroup of COL and found similar estimates of scaled $\rho$ between the BLS regions and background in one subgroup with low differentiation $\left(0.64-0.81\right.$ vs. $0.65, F_{3,7233}=2.25, P=$
0.080; Additional file 1: Table S10). To further evaluate the effects of recombination rate on genetic diversity, we simulated a variety of recombination rates from 0 to $250 \mathrm{cM} / \mathrm{Mb}$. We found that estimated diversities in all simulated data were significantly lower than the observations in the BLS regions ( $W$ ranges from 22,399,000 to $45,812,000, \quad P<2 \mathrm{e}^{-16}, \quad$ Mann-Whitney $U$ test; Additional file 1: Figure S7). These results suggest that the recombination rate might have a little effect on the patterns of nucleotide diversity in B. stricta. A previous study in $A$. thaliana also revealed that regions collinear to these BLS regions showed recombination rates close to the genome-wide average [38]. Third, we compared gene density (mean length of coding sequences per 20$\mathrm{kb}$ window) in outlier windows versus genome-wide. Gene density in two (LG1p and LG7p) of the BLS regions is slightly lower than the genomic background (3.93 kb vs. $4.56 \mathrm{~kb}, P<0.001$ for LG1p; $3.73 \mathrm{~kb}$ vs. 4.56 kb, $P=0.0016$ for LG7p; randomization test; Additional file 1: Table S9) but is not different from the genomic background in LG5p $(P=0.253$, randomization test; Additional file 1: Table S9). Additionally, controlling for recombination rate and gene density, the scaled diversity in the BLS regions is still higher than the genomic background $\left(F_{1,6217-6998}=307.12-1525.90, \quad P<\right.$ $2 \mathrm{e}^{-16}$; Additional file 1: Table $\left.\mathrm{S} 11\right)$. These results suggest that a high recombination rate or low gene density is unlikely to generate genomic islands of high diversity. Therefore, our results suggest that these regions reflect long-term balancing selection [10]. The hypothesis of long-term balancing selection was also supported by an excess of intermediate-frequency alleles (higher Tajima's $D$, and Fay and Wu's $H$ ) and lower population differentiation $\left(F_{S T}\right)$ in the BLS regions compared to the genomic background (Additional file 1: Table S9). In comparison with genome-wide averages, these regions showed higher absolute divergence $\left(d_{X Y}\right)$ and also relative node depth (RND [39]; Additional file 1: Table S9), which takes into account varied mutation rate across the genome by dividing $d_{X Y}$ of each group pair with their mean divergence to an outgroup species, $B$. retrofracta. These results may reflect higher levels of ancestral polymorphism prior to the split of these groups [11].

\section{Long-term balancing selection in disease resistance genes}

Plant disease resistance genes, such as nucleotide-binding site-leucine-rich repeat (NBS-LRR) gene family, can be targets of balancing selection, and genetic variation in these gene regions could be maintained for long periods by transient or frequency-dependent selection [40]. While NBSLRR genes have not been functionally characterized in $B$. stricta, previous studies have found several NBS-LRR genes under balancing selection in closely related species, e.g., $A$. thaliana [41, 42] and Capsella [43]. In the B. stricta 
genome [21], we identified 378 genes that were homologous to members of NBS-LRR gene family of A. thaliana [44], with $277(60 \%)$ of them located in the BLS regions-a sixfold enrichment of NBS-LRR in BLS regions (Additional file 1: Table S8). Strong positive correlations (Spearman's $\rho=0.19-0.41, P<0.001$; Additional file 1: Figure S8) between density of NBS-LRR genes and scaled diversity suggest long-term balancing selection on NBS-LRR genes and may have maintained genetic diversity in BLS regions. To avoid ambiguous alignments due to rapid evolution of NBS-LRR genes [44], we excluded NBS-LRR gene regions (4.8\% of the three genomic regions) from the data analysis. Genetic diversity in the BLS regions is elevated, even when NBS-LRR genes themselves have been removed (Additional file 1: Figure S9). Removing $\pi$-islands with lower coverage depth from BLS regions also did not change our conclusions (Additional file 1: Table S9). These results suggest that the high variation was not due to the readmapping errors in paralogous loci or highly polymorphic regions. Rather, historical balancing selection could act on NBS-LRR genes and maintain ancestral haplotypes with similar frequency in different geographical lineages of $B$. stricta. The high diversity in the BLS regions after excluding NBS-LRR genes suggested that intergenic regions linked to these genes were affected by selection. The average length of affected haplotypes is $\sim 72 \mathrm{~kb}(20 \mathrm{Mb}$ divided by 277 genes), much longer than the level of LD in this species (Additional file 1: Figure S5). Future studies, taking advantage of long-read sequencing technologies and functional genomic analyses, can reconstruct the ancient haplotypes and examine candidate selected genes.

An early population genomic study in A. thaliana found that regions syntenic to these BLS regions also show enrichment for NBS-LRR genes and elevated nucleotide polymorphism [45] (also see Additional file 1: Figure S10). This suggests that variation in the BLS regions may have been shaped by balancing selection over millions of years since these genera diverged [46].

\section{Sorting of ancient polymorphisms in divergence islands}

Balanced polymorphisms in ancestral populations could be maintained by long-term balancing selection during speciation, generating genomic regions with increased genetic diversity within and among daughter populations [10]. Also, ancient polymorphisms could be partitioned among descendant lineages, resulting in genomic regions with increased divergence. To look for genomic regions with elevated divergence among groups, we estimated Weir and Cockerham's weighted $F_{S T}$ in 20-kb nonoverlapping windows across the genome and computed $Z$-transformed $F_{S T}\left(Z-F_{S T}\right)$ scores separately in each group pair [3]. Because the BLS regions were under long-term balancing selection, they were excluded from the data analyses in this section. Genomic regions with
$Z-F_{S T} \geq 2$, corresponding to $3.04 \%$ of windows from all comparisons, were identified as outlier windows (" $F_{S T}$ islands"; Fig. 4). As previously found in other species [3$7]$, genetic divergence along the genome was highly heterogeneous and $F_{S T}$-islands scattered across the genome in all group pairs (Fig. 4; Additional file 1: Figure S11). As expected, increased genome-wide differentiation obscures genomic regions with elevated divergence [5]: we detected fewer $F_{S T}$-islands in comparisons of more divergent groups. For example, more than 300 islands were observed in comparisons among groups of EAST subspecies (e.g., COL, UTA, and NOR, $F_{S T}=0.12-0.21$ ), but only 22 in a comparison between sympatrically distributed WES and NOR groups (Additional file 1: Table S12), which showed the highest population differentiation $\left(F_{S T}=0.56\right.$; Additional file 1: Table S3). The coalescent simulation showed that the $F_{S T}$ values in genomic islands were significantly higher than the simulated results ( $W$ ranges from $1,188,900$ to $35,200,000, P<5 \mathrm{e}^{-16}$, Mann-Whitney $U$ test; Additional file 1: Figure S12), suggesting that neutral demographic processes cannot explain the elevated differentiation in outlier windows.

\section{Relative vs. absolute divergence in divergence islands}

To examine which factors have contributed to the formation of $F_{S T}$-islands [3, 11, 13], for each group pair, we compared the level of $d_{X Y}$ and RND in islands versus the genomic background. We found that both mean $d_{X Y}$ and mean $R N D$ were significantly higher in $F_{S T}$-islands of all group pairs $\left(P<10^{-5}\right.$, randomization test; Fig. 4; Additional file 1: Table S3), suggesting the elevated $d_{X Y}$ in genomic islands was not due to the increased substitution rates. Elevated levels of both relative divergence $\left(F_{S T}\right)$ and absolute divergence $\left(d_{X Y}\right)$ in $F_{S T}$-islands are compatible with a model in which these islands were derived from divergent sorting of ancient polymorphisms $[13,21]$. Consistent with this hypothesis, the coalescent time between the most divergent haplotypes in genomic islands was $\sim 1$ million years ago, which is much earlier than the split of B. stricta groups (Additional file 1: Figure S13). Alternatively, the $F_{S T}$-islands also could result from differential gene flow among genomic regions, i.e., restricted gene flow in islands versus high gene flow elsewhere in the genome [11]. Under this alternative hypothesis, $F_{S T}$-islands would be more pronounced in sympatric group pairs, since gene flow could be higher than for allopatric groups. In contrast, $d_{X Y}$ was significantly higher in $F_{S T}$-islands of both sympatric and allopatric group pairs, and the sympatric group pair (WES and NOR) showed the highest $F_{S T}$ and the lowest number of $F_{S T}$ islands among comparisons (Additional file 1: Tables S3 and S12). This indicates low gene flow between EAST and WEST subspecies in their zone of sympatry. Coalescent simulation supported a model with low gene flow 


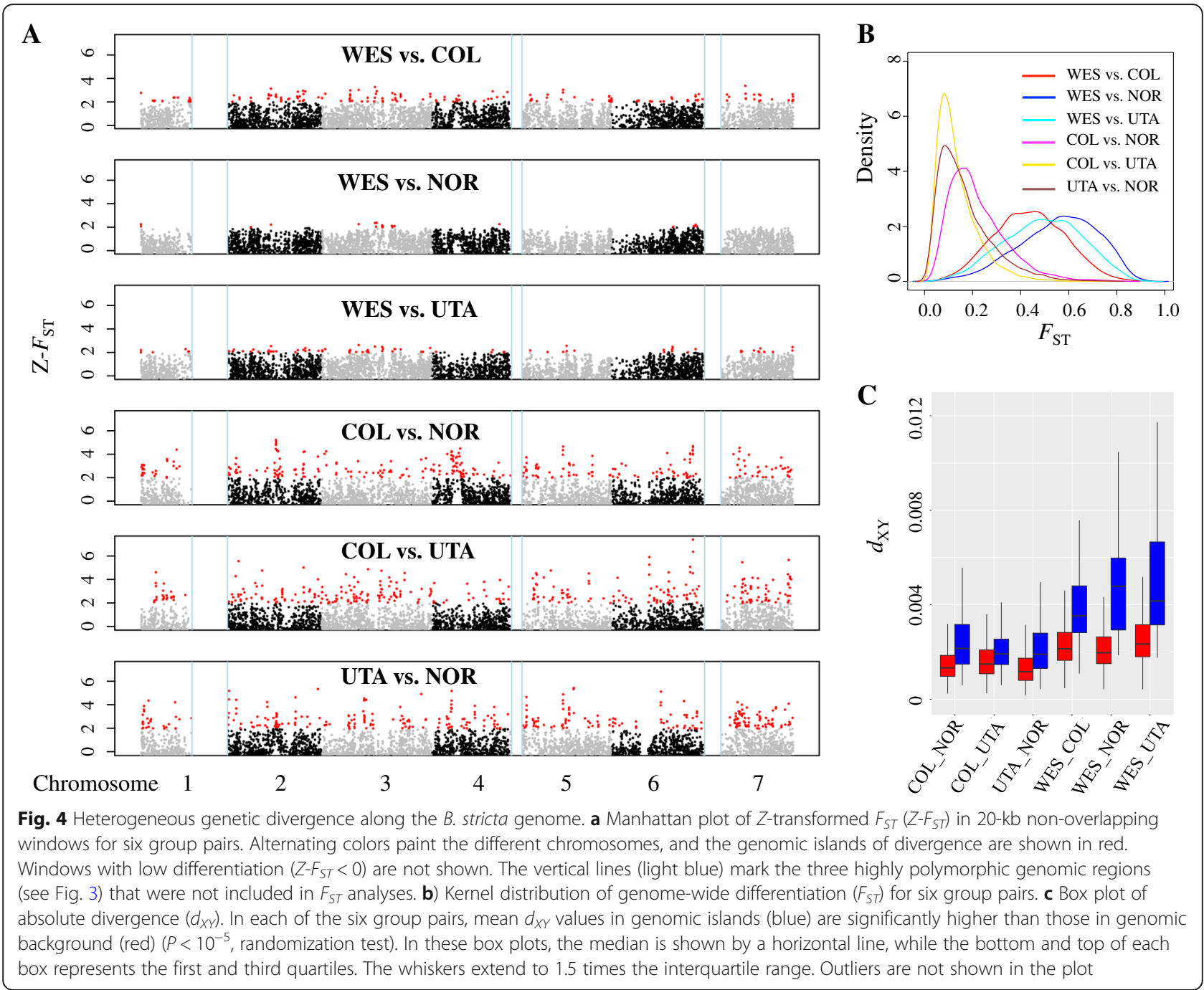

among the groups (Fig. 2; Additional file 1: Figure S4; Additional file 1: Table S6), in agreement with the high inbreeding coefficient [17] and low seed dispersal of Boechera [15]. The geographically isolated distribution of $B$. stricta groups (except WES and NOR) together with the low frequency of stricta $\times$ stricta admixed genotypes, also suggest that gene flow has been limited among the groups. Therefore, our observations indicate that reduced levels of recent gene flow are unlikely to be an important contributor to the formation of $F_{S T}$-islands in B. stricta.

We further tested whether $F_{S T}$-islands could be due to the ongoing background selection or recurrent selective sweeps. These two processes tend to reduce genetic polymorphism and to elevate $F_{S T}$ in lowrecombination regions [11], so we compared the recombination rate between $F_{S T}$-islands and the rest of the genome and tested the correlation between differentiation $\left(F_{S T}\right.$ and $\left.d_{X Y}\right)$ and recombination in $F_{S T^{-}}$ islands. Among the 12 comparisons (four group pairs, each containing two tests for two groups), only two comparisons showed a significant lower recombination in $F_{S T}$-islands than in background ( 0.668 vs. $1.037, P=$ 0.031 ; 0.718 vs. $0.901, P<0.001$; randomization test with Bonferroni correction; Additional file 1: Table S3) . Thus, $F_{S T}$ values in $F_{S T}$-islands are significantly higher than background after controlling for recombination and $/$ or gene density $\left(F_{1,6126-6820}=86.43-5123.27, P<\right.$ $2 \mathrm{e}^{-16}$; Additional file 1: Table S13). For regression tests, we found two significantly negative relationships out of 12 comparisons. It is possible that other historical factors, such as demographic history, might explain this pattern (Additional file 1: Figure S14). Additionally, if $F_{S T}$-islands were mainly produced by background selection or recurrent selective sweeps, $d_{X Y}$ within islands would be decreased or unaffected $[3,11]$, which is inconsistent with our observation of elevated $d_{X Y}$ in genomic islands. 


\section{Positive selection in divergence islands}

Within groups, $F_{S T}$-islands showed low nucleotide diversity $(\pi)$, excess of low frequency alleles (more negative Tajima's $D$ ) , and high-frequency derived alleles (more negative Fay and Wu's $H$ ) in one or both groups of each comparison $\left(P<10^{-5}\right.$, randomization test; see details in Additional file 1: Table S3), consistent with positive selection in these regions. It has been found that ancient polymorphisms under long-term balancing selection also could be under recent positive selection and promote adaption of humans to local environments [47]. To further infer possible functional influences, we conducted Gene Ontology (GO) analyses of these genomic islands. Comparisons showed that multiple GO categories with important metabolic processes and molecular functions (e.g., sucrose metabolic processes, catalytic activity) were overrepresented for genes located in genomic islands (Additional file 1: Table S14), suggesting a diverse set of genes and functional categories may have contributed to adaptive evolution of $B$. stricta.

\section{Conclusions}

We used population genomic analyses to track the divergence processes of $B$. stricta and to investigate the evolutionary forces that have shaped diversity within this species. We found that four genetic groups in B. stricta diverged during the Late Pleistocene. Our results suggest that long-term balancing selection on disease resistance genes may have maintained ancestral haplotypes across descendent lineages, resulting in elevated genetic diversity in three genomic regions comprising $10 \%$ of the genome. We further demonstrate that genomic regions with elevated divergence ( $F_{S T}$-islands) among the four lineages may be derived from divergent sorting of ancient polymorphisms, instead of heterogeneous gene flow or recurrent selective sweeps. These findings provide evidence that elevated genetic diversity due to balancing selection also may increase population differentiation by sorting balanced polymorphisms during divergence processes. This study highlights the importance of ancestral balanced polymorphisms as crucial constituents of genome-wide variation and incipient speciation.

\section{Methods}

\section{Sample collection, whole-genome sequencing, mapping, and SNP calling}

We analyzed 517 B. stricta accessions across the species range in the western USA (Fig. 1; Additional file 1: Table S1). These accessions are part of the B. stricta Reference Panel, available from the Arabidopsis Biological Resource Center. All accessions have the common, non-inverted haplotype on chromosome 1 [21]. Seeds were germinated and grown in the greenhouse for one generation to produce self-pollinated seeds for this study. Following the protocol of Lee et al. [21], we extracted genomic DNA of each accession from $\sim 0.1 \mathrm{~g}$ young leaf tissues using Qiagen DNeasy Plant Mini kits (Qiagen, Hilden, Germany) and measured the concentration using a Qubit fluorometer (Invitrogen, Carlsbad, CA, USA).

Paired-end sequencing libraries were prepared for each sample, and the sequencing was carried out on the Illumina HiSeq 2000/2500 platform at the Joint Genome Institute (JGI). Plate-based DNA library preparation for Illumina sequencing was performed on the PerkinElmer Sciclone NGS robotic liquid handling system using KAPA Biosystems Library Preparation Kit. About 200 ng DNA was sheared to $475-600$ bp using a Covaris LE220 Focused-ultrasonicator. The sheared DNA fragments were size selected by double-SPRI, and then the selected fragments were end-repaired, A-tailed, and ligated with Illumina compatible sequencing adaptors from IDT containing a unique molecular index barcode for each sample library. The prepared libraries were quantified using KAPA Biosystem's next-generation sequencing library qPCR kit and run on a Roche LightCycler 480 real-time PCR instrument. The quantified libraries were then multiplexed with other libraries, and the pool of libraries was then prepared for sequencing on the Illumina HiSeq sequencing platform utilizing a TruSeq paired-end cluster kit and Illumina's cBot instrument to generate a clustered flowcell for sequencing. Sequencing of the flowcell was performed on the Illumina HiSeq2000/2500 sequencer using a TruSeq SBS sequencing kit, following a $2 \times$ 100 or $2 \times 150$ indexed run recipe.

We used trimmomatic v0.36 [48] to remove adapter sequences and trim bases from both ends of reads when the base quality was $<30$. After trimming, reads $<36 \mathrm{bp}$ were discarded. We then aligned high-quality reads of each genotype to the $B$. stricta reference genome v1.2 [21] with BWA [49]. We used GATK v3.8 [25] for base quality recalibration, indel realignment, and simultaneous SNP and indel discovery via HaplotypeCaller. After that, genotypes (in gVCF files) of all individuals were joined together by using the default hard filtering parameters as prescribed by GATK v3.8 best practices. Only sites with mapping quality $\geq 30$ and base quality $\geq 30$ were considered for calling variants in HaplotypeCaller.

\section{SNP filtering and validation}

As some analyses required called genotypes, we used stringent filtering criteria to minimize false positives from SNP and genotype calls and further validated the accuracy of variant calling. We used the following filtering criteria: (1) homozygous genotypes were assigned as missing if supported by less than two reads; (2) heterozygous genotypes were assigned as missing if supported by less than 20 reads or reference ratio (number of reads supporting reference allele/number of reads supporting 
alternative allele) $<0.25$, or $>0.75$; and (3) SNPs were discarded if they met any one of the following criteria: genotyped in fewer than $50 \%$ of individuals, mean depth $>20$, more than one variant allele was observed, sites where the proportion of heterozygous genotypes was $>$ $15 \%$ (B. stricta is predominantly inbred; hence, high heterozygosity may indicate paralogous loci), or if reference or variant alleles were indels. We further removed 20 samples with missing rate $>0.60$, five duplicated samples from the same inbred family, six samples with divergent morphology or identified as outliers by PCA, and two samples without geographical information (Additional file 1: Table S1). Finally, we retained 4,125, 395 high-quality SNPs and 484 genotypes for the following analyses.

To gain insight into the variant calling and genotype accuracy, we compared individual genotypes from this re-sequencing dataset with genotypes from 129 loci (total $71 \mathrm{~kb}$ ) previously assayed by Sanger sequencing $[18,50]$. Twenty-one inbred lines sequenced by both Illumina and Sanger methods were used for this comparison (Additional file 1: Table S2). Each genotype of 21 individuals (sequenced at depth 2.7-5.8x) inferred by GATK was compared to the Sanger-sequenced ones from the same inbred lines. Genotypes from the resequencing data identical to the genotypes from the Sanger dataset were considered as true positives, and conflicting genotypes were considered as false. $99.89 \%$ of genotypes from the re-sequencing data are identical to genotypes from the Sanger dataset, indicating that high confidence SNPs were genotyped in our dataset.

To further monitor the accuracy of genotypes over different coverage depths, we downloaded reads of two genotypes that were sequenced at very high depth, $\sim 400 \times$ (Accession LTM, the genotype used for the reference genome) and $\sim 170 \times$ (SAD12, referred to as RP067 in this study) [21]. Next, we randomly downsampled these datasets to sub-datasets with depth from 1 to $40 \times$ (Additional file 1: Table S15). SNPs were called and filtered from these sub-datasets using the same pipelines as described above. For LTM, we compared genotype calls from sub-datasets with different depths to the reference genome. True positives (TP) are positions identical to the reference, false heterozygotes (FHET) are positions called as heterozygous genotypes (all sites are called as homozygotes in the reference genome), false homozygotes (FHOM) are positions called as homozygous genotypes but different from the reference, and missing SNPs (MISS) are non-genotyped sites. For SAD12, we compared genotypes called from each sub-dataset to those called from the highest depth data $(\sim 170 \times)$. True positive sites (TP) are defined as positions identical between low- and high-depth datasets; false heterozygotes (FHET) are positions different between datasets, with at least one heterozygote called; false homozygotes (FHOM) are positions called as different homozygotes between datasets; and missing SNPs (MISS) are sites genotyped in high-depth data but not called in low-depth data. For both LTM and SAD12, the true positive rate (TPR) is defined as $\mathrm{TP} /(\mathrm{TP}+\mathrm{FHET}+\mathrm{FHOM})$, false discovery rate (FR) is defined as (FHET + FHOM $) /(\mathrm{TP}+\mathrm{FHET}+\mathrm{FHOM})$, and missing rate (MR) is defined as MISS/(TP + FHET + FHOM + MISS). High TPR was found in LTM (99.9099.51\%) and SAD12 (98.75-99.82\%) datasets with different depths (Additional file 1: Table S15). It is not surprising to see high accuracy of called genotypes based on relative low sequencing depth $(5.05 \times)$ in $B$. stricta, because it is an inbreeding species with extremely low heterozygous rate [17, 21]. A similar result was reported in $A$. thaliana, another inbreeding species [32].

\section{Population structure}

To investigate population structure in B. stricta, we (1) conducted admixture analyses using Admixture v1.3.0 [27], FastSTRUCTURE v1.0 [28], sNMF [29], and NGSadmix [30]; (2) performed principal component analysis (PCA) with EIGENSOFT v6.0 [51]; and (3) constructed neighbor-joining (N) trees using MEGA v7 [52] with 1000 bootstrap samples. We ran Admixture, FastSTRUCTURE, sNMF, and NGSadmix with $K$ values ranging from 1 to 10 and repeated the process 20 times with different seeds. The best $K$ (i.e., the number of putative populations) was chosen by the $\Delta K$ method [53]. A tenfold cross-validation procedure and a cross-entropy criterion were also used for evaluating the runs with different $K$ values in Admixture and sNMF, respectively. Genotype likelihoods estimated by ANGSD [26] were used as input for NGSadmix, and genotypes called by GATK were used for other methods. For population structure analyses, we discarded SNPs with missing rate $>20 \%$ and minor allele frequency (MAF) $<5 \%$. We also excluded highly correlated SNPs by performing an LD-based SNP pruning process in PLINK v1.90 [54]. To do this, we scanned the genome with sliding windows of 50 SNPs in size, advancing in steps of five SNPs, and removed any SNP with a correlation coefficient $>0.2$ with any other SNP within the window. This yielded 27,765 independent SNPs for the analyses of population structure.

\section{Isolation by distance}

To investigate the pattern of isolation by distance in each group, we calculated genetic and geographic distances between each pair of genotypes and tested the correlation of genetic and geographic matrices by Mantel test with 10,000 permutations (permuting rows and columns) implemented in the R package VEGAN [55]. We further quantified the strength of the IBD in each group. To account for uneven sampling, we grouped genotype pairs into sequential $10 \mathrm{~km}$ 
bins (e.g., $0-10 \mathrm{~km}, \quad 10-20 \mathrm{~km}$ ) and calculated mean genetic distance from all genotype pairs in each bin. After that, we fit a weighted linear regression by considering the number of genotype pairs in each bin and calculated the slope and intercept of genetic distance against geographic distance. We discarded bins representing geographic distance less than $20 \mathrm{~km}$ or larger than $120 \mathrm{~km}$, due to a substantial deviation from the regression line. We also removed bins with less than 15 genotype pairs. The ratio of increase of pairwise diversity across geographic distance was estimated by dividing slope by mean pairwise diversity across all bins within $20-120 \mathrm{~km}$. We estimated the standard error of the ratio based on 1000 bootstraps. We also tried $20 \mathrm{~km}$ bins and got very similar results (data not shown). Ten isolated WES accessions from the Washington Cascades and the Sierra Nevada were excluded from IBD analyses, because they are located far from the distribution center of the WES group.

\section{Linkage disequilibrium}

We estimated genome-wide LD species-wide (484 individuals), as well as for each group (WES, COL, UTA, and NOR). We extracted common SNPs with MAF above 0.05 and calculated the mean-squared correlation $\left(r^{2}\right)$ for each pair of common SNPs within 50-kb windows using plink v1.90 [54]. The decay of LD with physical distance (bp) was estimated using nonlinear regression using Eq. 1 of Hill and Weir [56].

\section{Population demography Fastsimcoal2 simulation}

We inferred the demographic history of B. stricta by using a coalescent simulation-based method in fastsimcoal v.2.6.0.3 [33]. We tested fourteen demographic models (Additional file 1: Figure S3); all models contained four contemporary groups and began with the splitting of the two subspecies (WES vs. the ancestor of other three groups), followed by splitting of COL within the EAST subspecies, and the final split between UTA and NOR groups. These models differed with regard to (1) whether gene flow was present among groups and (2) how population size changed within groups (Additional file 1: Figure S3). Because missing data can lead to biased estimates of the site frequency spectrum (SFS), we performed a downsampling procedure following Thome and Carstens [57]. For each individual, we randomly chose one haplotype, since $B$. stricta is a largely inbreeding species. For each site, we resampled (without replacement) 39, 78, 63, and 52 genotypes from WES, COL, UTA, and NOR groups, respectively, to maximize the number of segregating SNPs. Sites were discarded if the sample size (nonmissing genotypes) was less than the threshold in any group. We excluded SNPs from three genomic regions under long-term balancing selection (see the "Balancing selection in B. stricta genomes" section) and only used fourfold degenerate sites and intergenic regions, because they are less affected by selection. Finally, 1,455,094 SNPs were retained to estimate SFS. To minimize biases when determining the ancestral allelic states, we generated folded SFS following the methods described by Excoffier et al. [33]. For each model, we performed 50 independent runs with 100,000 coalescent simulations as well as 10-40 conditional maximization algorithm cycles to find the global maximum-likelihood parameter estimates. The best model was chosen based on Akaike's weight of evidence following Excoffier et al. [33]. To obtain the 95\% confidence interval of the best model, we generated 100 parametric bootstraps and estimated the parameters on each bootstrap replicate using the same settings as for the analyses of the original dataset. Generation time of 2 years and mutation rate of $7 \times 10^{-9}$ substitutions per site per year in A. thaliana [34] were used to convert the model parameters to absolute values. To evaluate the goodnessof-fit of demographic models, we performed 100,000 coalescent simulations under the maximum likelihood estimates of population parameters, calculated expected SFS, and compared with the observed SFS. We also compared two summary statistics $\left(\pi\right.$ and $F_{S T}$ ) between simulated and observed data.

\section{Stairway plot analyses}

We applied the Stairway plot v2 [35] method with folded SFS (generated as for fastsimcoal2, above) to infer the historical changes of $N_{\mathrm{e}}$ over time in each genetic group. We used default settings to run Stairway, including $2 / 3$ of the data for training and four random break points at (nseq-2)/4, (nseq-2)/2, (nseq-2)*3/4, and nseq-2. As suggested by the authors, we created 200 input files using the provided script and estimated the median and 95\% confidence interval of demographic parameters based on these files. We converted estimates to absolute values based on a generation time of 2 years and a mutation rate of $7 \times 10^{-9}$ substitutions per site per year.

\section{Genome-wide scans for regions with elevated diversity and/or divergence}

We partitioned scaffolds into 20-kb windows, and calculated the sequence coverage by counting the number of available sites in each window. To obtain all available sites, we used the "-allSites" argument in GATK and filtered non-segregating sites using the same quality thresholds as for segregating sites (see the "SNP filtering and validation" section). For a window to be included in the downstream analyses, we required (1) at least 5000 sites left, after filtering steps; (2) at least $20 \mathrm{SNPs}$, for summary statistics based on segregating sites (e.g., Tajima's $D$ and $F_{S T}$ ); and (3) at least 2000 bases available from both ingroup and 
outgroup species, for outgroup statistics requiring outgroup information, such as Fay and Wu's $H$ and RND.

\section{Intra-population summary statistics}

For each group of B. stricta and the species as a whole, we estimated SFS and related population genetic statistics using a probabilistic method implemented in ANGSD v0.919 [26]. We filtered the data by (1) removing reads with a minimal mapping quality of 30 and bases with a minimal quality score of 30 (-minMapQ and $-\mathrm{min} Q$ ), (2) removing sites with information from less than $50 \%$ of individuals (-minInd), (3) removing sites with a $P$ value higher than $1 \times 10^{-4}$ (-snp_pval), (4) assigning genotypes as missing if the depth was less than two for an individual, and (5) removing sites that did not pass filtering criteria above (see the "SNP filtering and validation" section). We estimated per-individual inbreeding coefficients in ngsF-HMM [58] and incorporated them into the calculation of SFS in ANGSD. Using genotype likelihoods based on the GATK genotyping model [59], we estimated folded and unfolded SFS and derived a set of population genetic summary statistics in $20-\mathrm{kb}$ windows. We estimated nucleotide diversity $(\pi)$ and Tajima's $D$ on the basis of folded SFS and calculated Fay and Wu's $H$ from the unfolded SFS. We used $B$. retrofracta $[21,36]$ as the outgroup species to infer the ancestral allelic state to estimate the unfolded SFS.

\section{Inter-population summary statistics}

We used custom Python scripts to calculate the relative genetic differentiation (Weir and Cockerham's weighted $\left.F_{S T}\right)$ [60], absolute divergence $\left(d_{X Y}\right)$ [61], and net pairwise nucleotide divergence $\left(d_{a}\right)$ [61] for six pairwise comparisons among the four genetic groups (Fig. 1). To account for the variable mutation rate across the genome, we also estimated the relative node depth (RND) [39] by dividing $d_{X Y}$ of each group pair with their mean divergence to an outgroup species (B. retrofracta) [21, 36]. In each pairwise comparison, parameters were estimated on sites with at least $50 \%$ of individuals successfully genotyped per population. For $F_{S T}$, we calculated two variance components (the numerator and denominator) for each segregating site, averaged them separately, and obtained the window-based estimates as a "ratio of average" [60]. For $d_{X Y}$ and RND, we obtained windowbased values by averaging per-site estimates across all sites (both variable and monomorphic) passing the initial quality filters for each window.

\section{Recombination rate and gene density}

Population-scaled recombination rates $\left(\rho=4 N_{\mathrm{e}} \mathrm{c}\right)$ were estimated for each group using the program LDhelmet v.1.10 [37]. We ran LDhelmet with default parameters $(100,000$ burn-in iterations, 1000,000 Markov chain iterations, and a block plenty of 50) to estimate recombination rate between each pair of SNPs and then weight-averaged over each 20$\mathrm{kb}$ window. We only used SNPs with MAF $>5 \%$ to minimize the effects of rare variants, and only retained windows with at least 10 SNPs left after filtering. Using lookup tables with $\theta=0.001$ (close to estimates of genomic background) and $\theta=0.01$ (close to estimates in three genomic region with elevated diversity) yielded quantitatively identical results (Pearson's correlation coefficient $r=0.999$, $\left.P<2.2 \mathrm{e}^{-16}\right)$; thus, only the results based on $\theta=0.001$ were reported. To account for the influence of effective population size on estimated $\rho$, we divided $\rho$ by diversity $(\pi)$ in each 20-kb window following Wang et al. [4] and compared $\rho / \pi$ between islands and the rest of the genome. Gene density was estimated as the total length of coding sequences within each of the $20-\mathrm{kb}$ windows.

\section{Outlier screen}

To identify the genome regions with elevated diversity $(\pi)$, we standardized $\pi$ in each group for each window and identified high-diversity windows with $Z-\pi \geq 2$ ( $\pi$-island, corresponding to $4.30 \%$ of windows from all comparisons). Following the same procedure, we standardized per-window $F_{S T}$ in each pair of groups to a $Z$-score based on the formula $Z-F_{S T}=\left(F_{S T} \times F_{S T}{ }^{\prime}\right) /$ std- $F_{S T}$ [3], where $F_{S T}$ is a perwindow estimate, and $F_{S T}{ }^{\prime}$ and std- $F_{S T}$ are the mean and standard deviation of $F_{S T}$ across windows. We excluded the BLS regions from the analyses because they show evidence of long-term balancing selection and defined windows with $Z-F_{S T} \geq 2$ (corresponding to $3.04 \%$ of windows from all comparisons) as outliers ( $F_{S T}$-island). Based on 100,000 permutations, we compared genomic islands vs. genome-wide background for possible differences in multiple summary statistics, including recombination rate, diversity, and $F_{S T}$.

To test whether $\pi$-islands or $F_{S T}$-islands could be due to demographic processes solely, we first simulated 100 , 000 segments $(20 \mathrm{~kb}$ each, the same size as the windows used to scan the genome) using demographic parameters of the best model estimated by fastsimcoal2 (see the "Population demography" section for details). We performed simulations with different levels of recombination $(0,1,5,10,30,50,100$, and $250 \mathrm{cM} / \mathrm{Mb})$ covering a wide range of recombination rates in plants [62], and generated eight datasets in total. For each dataset, we estimated $\pi$ and $F_{S T}$ for groups and group pairs, respectively. Finally, we compared $\pi$ (for $\pi$-islands) and $F_{S T}$ (for $F_{S T}$-islands) in islands versus those from simulated data by using the Mann-Whitney $U$ test.

We further focused on $130 \pi$-islands shared among all groups. These islands showed higher diversity and stronger signatures of balancing selection than islands specific to particular group(s). These islands were clustered into three genomic regions. We merged shared islands that were separated by less than $1.5 \mathrm{Mb}$ into single genomic segments 
and delimited these three highly polymorphic genomic regions to $15.3-24.4 \mathrm{Mb}$ on the short arm of chromosome 1 (LG1p), $0.47-3.4 \mathrm{Mb}$ on the short arm of chromosome 5 (LG5p), and 0.45-5.1 Mb on the short arm of chromosome 7 (LG7p). To look for patterns of their homologous regions in A. thaliana genome, we used SyMAP v3.4 [63] to identify the collinear regions between B. stricta [21] and A. thaliana genomes [32] and further blasted sequences of the three B. stricta genomic regions onto the A. thaliana genome to delimit the homologous regions. To calculate genetic diversity in A. thaliana, we downloaded SNP data from http://1001genomes.org/. Of the 1135 sequenced accessions, we retained 972 non-relicts from the native range $[21,32]$. Genotypes supported by less than 2 reads were assigned as missing. SNPs with indels, more than two alleles, more than $50 \%$ missing data, or located on masked genomic regions were further excluded. We partitioned $A$. thaliana into $10-\mathrm{kb}, 20-\mathrm{kb}$, and 100-kb windows, calculated per-site nucleotide diversity $(\pi)$ using custom Python scripts, and obtained the window-based values by averaging per-site estimates in each window. To correct for variation in mutation rate among genomic regions, we divided diversity by divergence to the outgroup species, Arabidopsis lyrata.

\section{Gene ontology enrichment analyses}

We performed GO analyses to test whether any functional classes of genes were over-represented in $\pi$-islands or $F_{S T}$ islands. We first calculated $P$ values of Fisher's test and subsequently corrected $P$ values for multiple testing with Benjamini-Hochberg FDR [64]. GO terms with FDR < 0.05 were considered as significantly enriched. GO analyses were conducted with singular enrichment analysis in agriGO's Term Enrichment tool [65] and used B. stricta genome [21] as a reference.

\section{Additional file}

Additional file 1: Supplementary figures and tables. (PDF 8011 kb)

\section{Abbreviations}

ANOVA: Analyses of variance; BLS: Balancing selection; GO: Gene ontology; KYA: Thousand years ago; LD: Linkage disequilibrium; MAF: Minor allele frequency; NBS-LRR: Nucleotide-binding site-leucine-rich repeat; NJ: Neighbor joining; PCA: Principal component analysis

\section{Acknowledgements}

We thank the anonymous reviewers for the valuable comments on a previous draft, P. Ingvarsson and the Mitchell-Olds Lab for the helpful discussion, L. Excoffier for the help in the demographic analyses, and K. Ghattas for the greenhouse assistance.

\section{Authors' contributions}

$B W, J P M, N P, D S R, J S$, and TMO designed the research. NP, AS, CA, AL, and $\mathrm{KB}$ performed the research. JTL and TMO contributed new reagents and resources. BW, JPM, AL, and TMO analyzed the data. BW, JPM, NP, CRL, JTL, $\mathrm{KB}$, JS, and TMO wrote the paper. All authors read and approved the final manuscript.

\section{Funding}

BW was supported by the Guangdong Natural Science Funds for Distinguished Young Scholar (2018B030306040) and the Swedish Research Council (VR). TM-O was supported by grant R01 GM086496 from the National Institutes of Health (USA). Work conducted by the US Department of Energy Joint Genome Institute is supported by the Office of Science of the US Department of Energy under Contract No. DE-AC02-05CH11231. No funding body played a role in the study design or data collection, analysis, or interpretation.

\section{Availability of data and materials}

The short reads of each genotype have been deposited under GenBank accession numbers SRP054739, SRP134356, SRP134462, SRP134522, and SRP134640. All SNPs used in population genetic analyses, locations of all accessions, and custom scripts are available in the Dryad Data Archive at https://doi.org/10.5061/dryad.574pc6n [66]. Seeds from these accessions are available from the Arabidopsis Biological Resource Center.

\section{Ethics approval and consent to participate}

Not applicable

\section{Consent for publication}

Not applicable

\section{Competing interests}

The authors declare that they have no competing interests.

\section{Author details}

${ }^{1}$ Key Laboratory of Plant Resources Conservation and Sustainable Utilization, South China Botanical Garden, Chinese Academy of Sciences, Guangzhou 510650, China. '2Department of Biology, Duke University, Box 90338, Durham, NC 27708, USA. Institute of Ecology and Evolutionary Biology and Institute of Plant Biology, National Taiwan University, Taipei 10617, Taiwan, ROC.

${ }^{4}$ HudsonAlpha Institute for Biotechnology, Huntsville, AL 35806, USA.

${ }^{5}$ Department of Energy Joint Genome Institute, Walnut Creek, CA 94598, USA.

Received: 27 October 2018 Accepted: 4 June 2019

Published online: 21 June 2019

\section{References}

1. Seehausen O, Butlin RK, Keller I, Wagner CE, Boughman JW, Hohenlohe PA, Peichel CL, Saetre GP, Bank C, Brannstrom A, et al. Genomics and the origin of species. Nat Rev Genet. 2014;15:176-92.

2. Burri R, Nater A, Kawakami T, Mugal CF, Olason PI, Smeds L, Suh A, Dutoit L, Bures S, Garamszegi LZ, et al. Linked selection and recombination rate variation drive the evolution of the genomic landscape of differentiation across the speciation continuum of Ficedula flycatchers. Genome Res. 2015; 25:1656-65.

3. Han F, Lamichhaney S, Grant BR, Grant PR, Andersson L, Webster MT. Gene flow, ancient polymorphism, and ecological adaptation shape the genomic landscape of divergence among Darwin's finches. Genome Res. 2017;27:1004-15.

4. Wang J, Street NR, Scofield DG, Ingvarsson PK. Variation in linked selection and recombination drive genomic divergence during allopatric speciation of European and American aspens. Mol Biol Evol. 2016;33:1754-67.

5. Renaut S, Grassa CJ, Yeaman S, Moyers BT, Lai Z, Kane NC, Bowers JE, Burke $J M$, Rieseberg LH. Genomic islands of divergence are not affected by geography of speciation in sunflowers. Nat Commun. 2013;4:1827.

6. Vijay N, Bossu CM, Poelstra JW, Weissensteiner MH, Suh A, Kryukov AP, Wolf JBW. Evolution of heterogeneous genome differentiation across multiple contact zones in a crow species complex. Nat Commun. 2016;7:10.

7. Ma T, Wang K, Hu Q, Xi Z, Wan D, Wang Q, Feng J, Jiang D, Ahani H, Abbott RJ, et al. Ancient polymorphisms and divergence hitchhiking contribute to genomic islands of divergence within a poplar species complex. Proc Natl Acad Sci U S A. 2017;115:E236-43.

8. Malinsky M, Challis RJ, Tyers AM, Schiffels S, Terai Y, Ngatunga BP, Miska EA, Durbin R, Genner MJ, Turner GF. Genomic islands of speciation separate cichlid ecomorphs in an East African crater lake. Science. 2015;350:1493-8.

9. Wolf JBW, Ellegren $\mathrm{H}$. Making sense of genomic islands of differentiation in light of speciation. Nat Rev Genet. 2017;18:87-100. 
10. Charlesworth D. Balancing selection and its effects on sequences in nearby genome regions. PLoS Genet. 2006;2:379-84.

11. Cruickshank TE, Hahn MW. Reanalysis suggests that genomic islands of speciation are due to reduced diversity, not reduced gene flow. Mol Ecol. 2014;23:3133-57.

12. Ellegren H, Galtier N. Determinants of genetic diversity. Nat Rev Genet. 2016; 17:422-33.

13. Guerrero RF, Hahn MW. Speciation as a sieve for ancestral polymorphism. Mol Ecol. 2017;26:5362-8.

14. Nosil P, Funk DJ, Ortiz-Barrientos D. Divergent selection and heterogeneous genomic divergence. Mol Ecol. 2009;18:375-402.

15. Rushworth CA, Song B-H, Lee C-R, Mitchell-Olds T. Boechera, a model system for ecological genomics. Mol Ecol. 2011;20:4843-57.

16. Mitchell-Olds T. Arabidopsis thaliana and its wild relatives: a model system for ecology and evolution. Trends Ecol Evol. 2001;16:693-700.

17. Song BH, Clauss MJ, Pepper A, Mitchell-Olds T. Geographic patterns of microsatellite variation in Boechera stricta, a close relative of Arabidopsis. Mol Ecol. 2006;15:357-69.

18. Song BH, Windsor AJ, Schmid KJ, Ramos-Onsins S, Schranz ME, Heidel AJ, Mitchell-Olds T. Multilocus patterns of nucleotide diversity, population structure and linkage disequilibrium in Boechera stricta, a wild relative of Arabidopsis. Genetics. 2009;181:1021-33.

19. Lee CR, Mitchell-Olds T. Complex trait divergence contributes to environmental niche differentiation in ecological speciation of Boechera stricta. Mol Ecol. 2013;22:2204-17.

20. Lee CR, Mitchell-Olds T. Quantifying effects of environmental and geographical factors on patterns of genetic differentiation. Mol Ecol. 2011; 20:4631-42

21. Lee CR, Wang B, Mojica JP, Mandáková T, Prasad KVSK, Goicoechea JL, Perera N, Hellsten U, Hundley HN, Johnson J, et al. Young inversion with multiple linked QTLs under selection in a hybrid zone. Nat Ecol Evol. 2017;1: 0119.

22. Anderson JT, Lee CR, Mitchell-Olds T. Strong selection genome-wide enhances fitness tradeoffs across environments and episodes of selection. Evolution. 2014;68:16-31.

23. Anderson JT, Lee CR, Mitchell-Olds T. Life-history QTLs and natural selection on flowering time in Boechera stricta, a perennial relative of Arabidopsis. Evolution. 2011;65:771-87.

24. Prasad K, Song BH, Olson-Manning C, Anderson JT, Lee CR, Schranz ME, Windsor AJ, Clauss MJ, Manzaneda AJ, Naqvi I, et al. A gain-of-function polymorphism controlling complex traits and fitness in nature. Science. 2012;337:1081-4.

25. DePristo MA, Banks E, Poplin R, Garimella KV, Maguire JR, Hartl C, Philippakis AA, del Angel G, Rivas MA, Hanna M, et al. A framework for variation discovery and genotyping using next-generation DNA sequencing data. Nat Genet. 2011;43:491-8.

26. Korneliussen TS, Albrechtsen A, Nielsen R. ANGSD: analysis of next generation sequencing data. BMC Bioinformatics. 2014;15:356.

27. Alexander $\mathrm{DH}$, Novembre J, Lange K. Fast model-based estimation of ancestry in unrelated individuals. Genome Res. 2009;19:1655-64.

28. Raj A, Stephens M, Pritchard JK. fastSTRUCTURE: variational inference of population structure in large SNP data sets. Genetics. 2014;197:573-89.

29. Frichot E, Mathieu F, Trouillon T, Bouchard G, Francois O. Fast and efficient estimation of individual ancestry coefficients. Genetics. 2014;196:973-83.

30. Skotte L, Korneliussen TS, Albrechtsen A. Estimating individual admixture proportions from next generation sequencing data. Genetics. 2013;195:693-702.

31. Rosenberg NA, Mahajan S, Ramachandran S, Zhao CF, Pritchard JK, Feldman MW. Clines, clusters, and the effect of study design on the inference of human population structure. PLoS Genet. 2005;1:660-71.

32. 1001 Genomes Consortium. 1,135 genomes reveal the global pattern of polymorphism in Arabidopsis thaliana. Cell. 2016;166:481-91.

33. Excoffier L, Dupanloup I, Huerta-Sanchez E, Sousa VC, Foll M. Robust demographic inference from genomic and SNP data. PLoS Genet. 2013;9: e1003905.

34. Ossowski S, Schneeberger K, Lucas-Lledo Jl, Warthmann N, Clark RM, Shaw RG, Weigel $D$, Lynch $M$. The rate and molecular spectrum of spontaneous mutations in Arabidopsis thaliana. Science. 2010;327:92-4.

35. Liu XM, Fu YX. Exploring population size changes using SNP frequency spectra. Nat Genet. 2015;47:555-9.

36. Kliver S, Rayko M, Komissarov A, Bakin E, Zhernakova D, Prasad KVSK, Rushworth C, Baskar R, Smetanin D, Schmutz J, et al. Assembly of the
Boechera retrofracta genome and evolutionary analysis of apomixisassociated genes. Genes. 2018;9:185.

37. Chan AH, Jenkins PA, Song YS. Genome-wide fine-scale recombination rate variation in Drosophila melanogaster. PLoS Genet. 2012;8:e1003090.

38. Choi K, Reinhard C, Serra H, Ziolkowski PA, Underwood CJ, Zhao XH, Hardcastle TJ, Yelina NE, Griffin C, Jackson M, et al. Recombination rate heterogeneity within Arabidopsis disease resistance genes. PLoS Genet. 2016;12:30.

39. Feder JL, Xie XF, Rull J, Velez S, Forbes A, Leung B, Dambroski H, Filchak KE, Aluja M. Mayr, Dobzhansky, and Bush and the complexities of sympatric speciation in Rhagoletis. Proc Natl Acad Sci U S A. 2005;102:6573-80.

40. Fijarczyk A, Babik W. Detecting balancing selection in genomes: limits and prospects. Mol Ecol. 2015:24:3529-45.

41. Bakker EG, Toomajian C, Kreitman M, Bergelson J. A genome-wide survey of $R$ gene polymorphisms in Arabidopsis. Plant Cell. 2006;18:1803-18.

42. Karasov TL, Kniskern JM, Gao LP, DeYoung BJ, Ding J, Dubiella U, Lastra RO, Nallu S, Roux F, Innes RW, et al. The long-term maintenance of a resistance polymorphism through diffuse interactions. Nature. 2014;512:436-40.

43. Sicard A, Kappel C, Josephs EB, Lee YW, Marona C, Stinchcombe JR, Wright $\mathrm{SI}$, Lenhard M. Divergent sorting of a balanced ancestral polymorphism underlies the establishment of gene-flow barriers in Capsella. Nat Commun. 2015;6:7960.

44. Guo YL, Fitz J, Schneeberger K, Ossowski S, Cao J, Weigel D. Genome-wide comparison of nucleotide-binding site-leucine-rich repeat-encoding genes in Arabidopsis. Plant Physiol. 2011;157:757-69.

45. Clark RM, Schweikert G, Toomajian C, Ossowski S, Zeller G, Shinn P Warthmann N, Hu TT, Fu G, Hinds DA, et al. Common sequence polymorphisms shaping genetic diversity in Arabidopsis thaliana. Science. 2007:317:338-42.

46. Huang $\mathrm{CH}$, Sun R, Hu Y, Zeng L, Zhang N, Cai L, Zhang Q, Koch MA, AlShehbaz I, Edger PP, et al. Resolution of Brassicaceae phylogeny using nuclear genes uncovers nested radiations and supports convergent morphological evolution. Mol Biol Evol. 2016;33:394-412.

47. de Filippo C, Key FM, Ghirotto S, Benazzo A, Meneu JR, Weihmann A, Parra G, Green ED, Andres AM, Program NCS. Recent selection changes in human genes under long-term balancing selection. Mol Biol Evol. 2016;33:1435-47.

48. Bolger AM, Lohse M, Usadel B. Trimmomatic: a flexible trimmer for Illumina sequence data. Bioinformatics. 2014;30:2114-20.

49. Li H. Aligning sequence reads, clone sequences and assembly contigs with BWA-MEM. Preprint at https://arxiv.org/abs/1303.3997. Accessed 16 Mar 2013.

50. Gossmann TI, Song BH, Windsor AJ, Mitchell-Olds T, Dixon CJ, Kapralov MV, Filatov DA, Eyre-Walker A. Genome wide analyses reveal little evidence for adaptive evolution in many plant species. Mol Biol Evol. 2010;27:1822-32.

51. Patterson N, Price AL, Reich D. Population structure and eigenanalysis. PLoS Genet. 2006;2:2074-93.

52. Kumar S, Stecher G, Tamura K. MEGA7: molecular evolutionary genetics analysis version 7.0 for bigger datasets. Mol Biol Evol. 2016;33:1870-4.

53. Evanno G, Regnaut S, Goudet J. Detecting the number of clusters of individuals using the software STRUCTURE: a simulation study. Mol Ecol. 2005;14:2611-20.

54. Purcell S, Neale B, Todd-Brown K, Thomas L, Ferreira MAR, Bender D, Maller J, Sklar P, de Bakker PIW, Daly MJ, Sham PC. PLINK: a tool set for wholegenome association and population-based linkage analyses. Am J Hum Genet. 2007:81:559-75.

55. Oksanen J, Blanchet FG, Friendly M, Kindt R, Legendre P, McGlinn D, Minchin $\mathrm{P}, \mathrm{O}^{\prime}$ Hara R, Simpson G, Solymos $\mathrm{P}$, et al. vegan: community ecology package. R package version 24-1. 2016.

56. Hill WG, Weir BS. Variances and covariances of squared linkage disequilibria in finite populations. Theor Popul Biol. 1988;33:54-78.

57. Thome MTC, Carstens BC. Phylogeographic model selection leads to insight into the evolutionary history of four-eyed frogs. Proc Natl Acad Sci U S A. 2016;113:8010-7.

58. Vieira FG, Albrechtsen A, Nielsen R. Estimating IBD tracts from low coverage NGS data. Bioinformatics. 2016;32:2096-102.

59. McKenna A, Hanna M, Banks E, Sivachenko A, Cibulskis K, Kernytsky A, Garimella K, Altshuler D, Gabriel S, Daly M, DePristo MA. The genome analysis toolkit: a MapReduce framework for analyzing next-generation DNA sequencing data. Genome Res. 2010;20:1297-303.

60. Weir BS, Cockerham CC. Estimating F-statistics for the analysis of population structure. Evolution. 1984;38:1358-70.

61. Nei M. Molecular evolutionary genetics. New York: Columbia University Press; 1987. 
62. Choi $\mathrm{K}$, Henderson IR. Meiotic recombination hotspots - a comparative view. Plant J. 2015;83:52-61.

63. Soderlund C, Bomhoff M, Nelson WM. SyMAP v3.4: a turnkey synteny system with application to plant genomes. Nucleic Acids Res. 2011;39:e68.

64. Benjamini Y, Hochberg Y. Controlling the false discovery rate: a practical and powerful approach to multiple testing. J R Stat Soc Series B Stat Methodol. 1995;57:289-300

65. Tian T, Liu Y, Yan HY, You Q, Yi X, Du Z, Xu WY, Su Z. agriGO v2.0: a GO analysis toolkit for the agricultural community, 2017 update. Nucleic Acids Res. 2017;45:W122-9.

66. Wang B, Mojica JP, Perera N, Lee CR, Lovell JT, Sharma A, Adam C, Lipzen A Barry K, Rokhsar DS, Schmutz J, Mitchell-Olds T. Ancient polymorphisms contribute to genome-wide variation by long-term balancing selection and divergent sorting in Boechera stricta. Datasets SNPS, Samples and Scripts; 2019. https://doi.org/10.5061/dryad.574pc6n.

\section{Publisher's Note}

Springer Nature remains neutral with regard to jurisdictional claims in published maps and institutional affiliations.

Ready to submit your research? Choose BMC and benefit from:

- fast, convenient online submission

- thorough peer review by experienced researchers in your field

- rapid publication on acceptance

- support for research data, including large and complex data types

- gold Open Access which fosters wider collaboration and increased citations

- maximum visibility for your research: over $100 \mathrm{M}$ website views per year

At BMC, research is always in progress.

Learn more biomedcentral.com/submissions 